\title{
"Safety" technology: a hidden cause of diabetic ketoacidosis
}

\author{
Catherine H.Y. Yu MD MHSc
}

1 74-year-old woman with type 2 diabetes was transferred from a long-term care facility to an acute care hospital because of a three-day history of nausea, vomiting and decreased oral intake. She denied any other symptoms. Fourteen days earlier, she had been transferred to the long-term care facility from a rehabilitation facility where she had been residing for six months while recovering from a stroke. Her medications included insulin glargine and insulin aspart. At the emergency department, her heart rate was 135 beats/min, blood pressure was $100 / 89 \mathrm{~mm} \mathrm{Hg}$, respiratory rate was 18 breaths/min, oxygen saturation was $98 \%$ on room air and temperature was $36.4^{\circ} \mathrm{C}$. Physical examination showed neurologic findings of her previous stroke and no focus of infection. Results of laboratory testing included a blood glucose level of 36.0 (normal 4.0-7.8) $\mathrm{mmol} / \mathrm{L}$, an anion gap of 17 (normal 8-16) $\mathrm{mmol} / \mathrm{L}$ and positive serum ketones. Investigations did not identify infection or ischemia. Diabetic ketoacidosis was diagnosed. The patient received treatment in the intensive care unit with intravenous insulin and fluid for six hours, with prompt resolution of the diabetic ketoacidosis. She was discharged with a $42 \%$ increase in her daily dose of insulin. A precipitating cause for diabetic ketoacidosis was not identified, and the condition was concluded to be secondary to suboptimal insulin dosing.

Six days later, the patient was admitted to the intensive care unit of another acute care hospital with diabetic ketoacidosis. A complete work-up did not identify a precipitant.

One month later the patient was assessed at a diabetes clinic for a review of diabetes management. Despite consistent intake of carbohydrates and physical activity, the patient's capillary blood glucose measurements had been fluctuating widely from $2.2 \mathrm{mmol} / \mathrm{L}$ to "high" with no discernible temporal pattern. Her long-term care facility was administering insulin using safety pen needles with a sliding sleeve encircling the needle that is retracted by pressure on the injection site. The health care team thought the patient's soft, redundant abdominal tissue might be impeding the retraction of the pen's sleeve, and this was confirmed by trying to inject insulin into her abdomen during the appointment. The patient was given a box of nonretractable pen needles, and follow-up by telephone five days later confirmed the resolution of extreme glucose fluctuations.

Twenty days later, the patient presented again with diabetic ketoacidosis to an acute care hospital. Laboratory investigations did not identify a precipitant. Three days before this admission, the long-term care facility had exhausted the box of nonretractable pen needles and resumed use of pen needles with a retractable safety shield. The diabetic ketoacidosis resolved promptly with intravenous fluid and insulin. Further education was provided, including a team outreach visit and instructions on the use of nonretractable pen needles and standard insulin syringes.

\section{Discussion}

Diabetic ketoacidosis accounts for an estimated 115000 hospital discharges per year in the United States ${ }^{1}$ and is associated with a mortality rate of up to $6.1 \% .^{2}$ Precipitating causes must be identified and addressed to reduce mortality related to diabetic ketoacidosis. ${ }^{2}$ Infection is the most common precipitant for diabetic ketoacidosis. Other precipitating factors include acute illness (e.g., myocardial infarction), medications and inadequate insulin therapy. ${ }^{3}$ In this patient, a delay in identifying the underlying cause - inadequate insulin delivery — resulted in multiple admissions to hospital.

\section{- KeY POINTS}

- Failure to identify and manage the precipitating cause of diabetic ketoacidosis is the most common cause of mortality in this condition.

- Although infection is the most common precipitant of diabetic ketoacidosis, other factors, such as ischemia, medications and inadequate delivery of insulin, should be considered.

- Care should be taken when administering insulin with shielded safety needles to patients with soft, redundant skin at injection sites. 
Shielded safety needles in diabetes

Needles with a retractable safety shield are primarily used in hospitals and long-term care facilities. The retractable shield was designed to reduce the risk of accidental puncture, eliminate reuse of needles and help patients overcome anxiety about needles. ${ }^{4}$ These needles feature an automatic shield (plastic cannula) that retracts on application of pressure onto the skin and hides the needle before, during and after injection. However, if incorrectly applied (particularly in the setting of soft, redundant skin), it is possible for the insulin to be deployed while the needle is outside the body. The automatic shield may prevent the user from seeing if the needle is subcutaneous. Thus, users must be educated on appropriate administration, which includes perpendicular insertion of the needle into the subcutaneous tissue or injection into a lifted skin fold to ensure a more firm surface.

\section{Safety and efficacy of shielded safety needles}

The insulin pen needle is the most common device resulting in needlestick injuries in nonhospital settings such as long-term care facilities, with $81 \%$ of needlestick injuries occurring during recapping of the insulin pen needle for disposal or reuse. ${ }^{5}$ The US and most Canadian provinces have passed legislation requiring that all health care workplaces use safety-engineered devices when feasible. ${ }^{6,7}$

Data regarding the effectiveness of safetyengineered devices are limited to industry-sponsored studies of device performance and user satisfaction, and small observational studies. In one study, 123 nurses were asked to use needles with a retractable shield in clinical practice for four weeks. ${ }^{8}$ They found that there were no needlestick injuries (out of 7854 injections) with the retractable needles, whereas there was one needlestick injury (out of 4491 injections) with use of regular needles. Eighty percent of nurses preferred the needles with retractable safety shields, citing personal safety. Of note, $7.7 \%$ of nurses commented that they could not evaluate the insulin dose injected with the retractable needles because the needle was covered by the plastic shield. This observation has been corroborated in a survey of 36 nurses piloting needles with retractable safety shields; only $39 \%$ were confident that the correct insulin dose was administered. In addition, three nurses indicated that they saw insulin on patients' skin after an injection. ${ }^{910}$ To date, there has been one report of a needlestick injury from an insulin pen with a retractable safety shield during a four-month pilot study. ${ }^{11}$
Although legislation mandates the use of safety-engineered needles in most clinical situations, conventional needles may be used in situations in which the safety-engineered needle may impair the effectiveness of treatment or result in harm. ${ }^{67}$ In these situations, other options include insulin syringes or self-administration by patients, concurrent with the implementation of safe work procedures, such as the use of adapted sharps disposal containers.

\section{References}

1. Centers for Disease Control and Prevention. Diabetes surveillance system. DKA as first-listed diagnosis for hospitalization. Atlanta (GA): Centers for Disease Control and Prevention; 2005.

2. Chen HF, Wang CY, Lee HY, et al. Short-term case fatality rate and associated factors among inpatients with diabetic ketoacidosis and hyperglycemic hyperosmolar state: a hospital-based analysis over a 15-year period. Intern Med 2010;49:729-37.

3. Kitabchi AE, Umpierrez GE, Miles JM, et al. Hyperglycemic crises in adult patients with diabetes. Diabetes Care 2009;32:1335-43.

4. Novofine Autocover [homepage]. Mississauga (ON) NovoNordisk; 2011. Available: www.novonordisk.com/diabetes /public/needles/novofine_autocover/default.asp (accessed 2011 July 15$)$.

5. Kiss P, De Meester M, Braeckman L. Needlestick injuries in nursing homes: the prominent role of insulin pens. Infect Control Hosp Epidemiol 2008;29:1192-4.

6. US Needlestick Safety and Prevention Act, HR 5178, 106th Cong, 2nd Sess, 2000.

7. Ontario Occupational Health and Safety Act, Ontario Regulation 474/07, 2007.

8. Lautier O, Mosnier-Podar H, Durain D, et al. Risk of needlestick injuries among nurses using NovoFine Autocover safety needles and nurses' satisfaction with the needles: The NOVAC Study. Excerpta Medica 2008;3:232-7.

9. Sheldon KA, Tubbs C. Implementation of insulin pens at an academic medical center. International Pharmaceutical Abstracts ASHP Midyear Clinical Meeting; 2007 Dec. 2; Las Vegas, Nevada.

10. Davis EM, Bebee A, Crawford L, et al. Nurse satisfaction using insulin pens in hospitalized patients. Diabetes Educ 2009;35: 799-809.

11. Bahl V, Antinopj-Lent K. Transitioning from patient-specific insulin vials to patient-specific insulin pens at the bedside: Quality improvement or not? Infect Control Hosp Epidemiol 2001; 22:456-8.

Affiliations: From the Division of Endocrinology and Metabolism, Department of Medicine, Faculty of Medicine, and the Keenan Research Centre, Li Ka Shing Knowledge Institute, St. Michael's Hospital, University of Toronto, Toronto, Ont.

The section Cases presents brief case reports that convey clear, practical lessons. Preference is given to common presentations of important rare conditions, and important unusual presentations of common problems. Articles start with a case presentation (500 words maximum), and a discussion of the underlying condition follows (1000 words maximum). Visual elements (e.g., tables of the differential diagnosis, clinical features or diagnostic approach) are encouraged. Written consent from patients for publication of their story is a necessity and should accompany submissions. See information for authors at www.cmaj.ca. 